\title{
MULTISCALE MODELING OF WEAR DEGRADATION IN CYLINDER LINERS*
}

\author{
ALVARO MORAES ${ }^{\dagger}$, FABRIZIO RUGGERI ${ }^{\ddagger}$, RAÚL TEMPONE $^{\dagger}$, AND \\ PEDRO VILANOVA ${ }^{\dagger}$
}

\begin{abstract}
Every mechanical system is naturally subjected to some kind of wear process that, at some point, will cause failure in the system if no monitoring or treatment process is applied. Since failures often lead to high economical costs, it is essential both to predict and to avoid them. To achieve this, a monitoring system of the wear level should be implemented to decrease the risk of failure. In this work, we take a first step into the development of a multiscale indirect inference methodology for state-dependent Markovian pure jump processes. This allows us to model the evolution of the wear level and to identify when the system reaches some critical level that triggers a maintenance response. Since the likelihood function of a discretely observed pure jump process does not have an expression that is simple enough for standard nonsampling optimization methods, we approximate this likelihood by expressions from upscaled models of the data. We use the Master Equation (ME) to assess the goodness-of-fit and to compute the distribution of the hitting time to the critical level.
\end{abstract}

Key words. pure jump processes, multiscale approximation, indirect inference, wear processes, Master Equation

AMS subject classifications. 60J75, 60J27, 62M05, 90B25, 60K10

DOI. $10.1137 / 130927024$

1. Introduction. It is well known that one of the main factors in the failure of heavy-duty diesel engines used for marine propulsion is wear of the cylinder liner [6]. The stochastic modeling of the wear degradation of cylinder liners is extensively treated in $[5,6,7,8]$ and the references therein. This wear process, at some point, will cause failure if no maintenance program is utilized. An effective maintenance program is one that can be carried out when there is some identifiable warning of the occurrence of failure. Then, preventive maintenance can be carried out on the basis of the current condition of the liner, generally when the maximum wear approaches a specified limit as imposed by warranty clauses.

In this work, we aim to use a multiscale indirect inference approach for the wear degradation problem. In our context, the term indirect inference is used in the sense that "it is impossible to efficiently estimate the parameters of interest because of the intractability of the likelihood function" [9]. But, instead of using a sampling-oriented method to obtain consistent estimators, we propose the use of a multiscale hierarchy of approximate "tractable" likelihoods. After optimizing these likelihoods, we plug the estimated parameters into our base model and assess its quality by checking confidence bands computed directly from the estimated base model.

The data set, $\mathbf{w}=\left\{w_{i}\right\}_{i=1}^{n}$, taken from [8], consists of wear levels observed on

*Received by the editors July 1, 2013; accepted for publication (in revised form) December 17, 2013; published electronically March 20, 2014. The first, third, and fourth authors are members of the SRI Center for Uncertainty Quantification in Computational Science and Engineering at KAUST. This research was performed when the second author visited KAUST.

http://www.siam.org/journals/mms/12-1/92702.html

${ }^{\dagger}$ Mathematical and Computer Sciences and Engineering Division, King Abdullah University of Science and Technology (KAUST), Thuwal, Saudi Arabia (alvaro.moraesgutierrez@kaust.edu.sa, raul. tempone@kaust.edu.sa, pedro.guerra@kaust.edu.sa).

${ }^{\ddagger}$ Consiglio Nazionale delle Ricerche, Istituto di Matematica Applicata e Tecnologie Informatiche, Milano, Italy (fabrizio@mi.imati.cnr.it). 
$n=32$ cylinder liners of eight-cylinder SULZER engines as measured by a caliper with a precision of $0.05 \mathrm{~mm}$. Warranty clauses specify that, to avoid failures, the liner should be changed before it accumulates a wear level of $4.0 \mathrm{~mm}$. Data are presented in Figure 1.

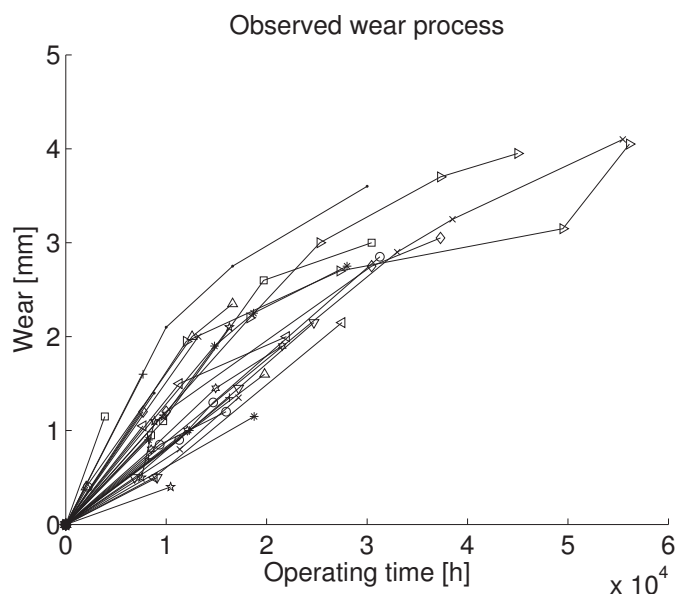

FIG. 1. Data set from [8]. Data refer to cylinder liners used in ships of the Grimaldi Group.

As a consequence of the finite resolution of the caliper, the set of possible measurements of the cylinder wear is represented with a finite lattice in the positive real line. For that reason, we propose to model the resulting measurements of the wear process as a Markovian pure jump process [10], which is the simplest class of pure jump processes. This type of process can be characterized by a finite set of possible jumps, each one having a certain intensity function (see section 2.1 for details).

In this work, we propose a multiscale inference approach that gradually allows us to estimate the number of possible jumps of the process, its amplitudes, and the corresponding intensity functions. We depart from the simplest possible pure jump model, i.e., the one that has only one possible jump with a linear intensity function, and proceed with more complex models by, for instance, adding more possible jumps and/or more general intensity functions.

Our base model, which defines the microscopic scale, is a continuous-time Markov pure jump process in a lattice. Since the process is observed only in a finite set of times, i.e., it is partially observed, its likelihood function usually cannot be written in a simple closed form amenable to performing standard optimization procedures. Proper inference based on partially observed continuous-time Markov chains in lattices should be based on likelihoods corresponding to nonhomogeneous Poisson processes. We refer the reader to the chapter "Inference for Stochastic Kinetic Models" in [14] for details on the mentioned likelihoods and on Monte Carlo Markov chain (MCMC) techniques for inference on pure jump processes. In [14], the inference procedure is intended only for linear rates and is based on MCMC and exact path-simulation techniques such as the stochastic simulation algorithm (SSA) by [4]. As a consequence, this inference methodology may be very computationally demanding and does not address the problem for general nonlinear rates. To the best of the authors' knowledge, there are no computationally low-cost sampling schemes from nonhomogeneous Poissonian bridges.

For that reason, the idea is to consider upscaled auxiliary versions for modeling 
our data, from which we can obtain simpler likelihood functions. Once we estimate the parameters from this approximate likelihood, we plug them into the base model and look at how well it fits the data. We use confidence bands, computed from the Master Equation (ME) [13] at the microscopic level, as a visual goodness-of-fit criteria. The inference methodology, motivated by the introduction of several temporal scales, is as follows; the first two categories correspond to macroscale indirect inferences, whereas the third category corresponds to the mesoscale indirect inferences, and the last corresponds to direct inference at the microscale level:

Perturbed mean field. We first approximate the likelihood function of our base microscopic model by the likelihood corresponding to its mean field (macroscale reaction rate ordinary differential equations (ODEs)) whose observations are perturbed with Gaussian noise.

Perturbed Gaussian process. If the parameters estimated for each member of a suitable family of macroscopic mean field models do not fit the experimental data, we translate the indirect inference problem into a slightly more complex one. In this case, the approximated likelihood corresponds to a Gaussian process whose mean and variance are obtained by a second-order moment expansion of the base model, perturbed with additive independent Gaussian noise. Then, provided that the probability distribution of the underlying pure jump process is unimodal for all times, we expect good agreement between the estimated microscopic model and the experimental data.

Langevin diffusion. If the estimated microscopic model does not fit the data, we translate the inference problem into the mesoscopic scale, where inference techniques for Langevin diffusions apply. We observe that the likelihood functions based on Gaussian models for the data are more restrictive than the likelihoods based on the Langevin one, because the latter allow us to model the time evolution of multimodal distributions. When a Gaussian model is appropriate, however, it is estimated much more quickly than a Langevin one.

Direct inference. In the same way, if the parameters estimated in a suitable family of mesoscale Langevin models do not fit the experimental data, we should make a direct inference at the microscopic level. It is worth mentioning that inference procedures at the mesoscale and microscale are much more involved from the computational point of view. For these two scales, the likelihood functions in general cannot be written in a closed form, and for that reason, they have to be approximated and optimized by sampling procedures [2].

In the literature there are other approaches for the wear inference problem based on pure jump processes that do not deal directly with the continuous-time model. For example, in [6], in the chapter "Stochastic Processes for Modeling the Wear of Marine Engine Cylinder Liners" the authors modeled the wear process as a continuoustime state-dependent Markov chain. In their methodology they approximated the continuous nature of time by using a discrete time Markov chain with uniform time steps and modeled its transitions probabilities with a Poisson kernel depending on two parameters. The resulting fitting with this approach is poor and the authors do not proceed further in this direction. In [8], a state-dependent, inhomogeneous in time Markov chain is proposed. The authors use a similar inference strategy by discretizing time and space. These approximation steps and their relevant associated errors are not discussed, and the computational cost of this approximate inference method explodes as one refines the time and/or space discretizations. 
Similar observations can be made for diffusion processes, where the lattice is approximated by a continuum of states and the Poissonian noise is replaced by Gaussian noise. Sampling bridges from diffusions is still an ongoing research area; see [1] and the references therein.

Our main contribution is twofold. First, we offer a novel approach to the problem of modeling the wear degradation of cylinder liners by using a continuous-time Markov chain in a lattice determined by caliper precision. Second, we take a first step toward a general methodology for a multiscale indirect inference approach. It is a first step because, for this particular problem, we did not need to use the mesoscopic or microscopic level of approximation.

The goals of this work are (i) to estimate the parameters of the wear process, modeled as a Markovian pure jump process and (ii) to obtain the distribution of the hitting time to the critical warranty level.

The remainder of this paper is organized as follows. Section 2 presents the base model and its upscalings and the system of ODEs for the first two moments of the base model. In section 3 , we present the model that actually fits the data. In section 4 , we derive the likelihood functions for the macroscopic scales. In section 5, we develop a method for computing the hitting time to the critical level based on the solution of the ME. Section 6 contains the results of the inference process and the distribution of the hitting time for the fitted model. Finally, section 7 offers the conclusions.

2. Methodology. In this section, we first present the elements of the base microscopic model and its infinitesimal generator. Then, we derive the macroscopic mean field and the mesoscopic Langevin approximations. Finally, we show how to derive a system of ODEs for the time evolution of the first two moments of the base model. The mean field and the second-order expansion are used in section 4 as the basis of the indirect inference method.

2.1. The pure jump process. Consider a Markov pure jump process, $X$, taking values in a lattice, $\mathcal{S}$, in $\mathbb{R}_{+}$. This means that the evolution of the state vector, $X(t)$, is modeled as a continuous-time Markov chain (see [11]).

Assume that each possible jump in the system occurs according to one of the pairs $\left\{\left(a_{j}(x ; \theta), \nu_{j}\right)\right\}_{j=1}^{J}$, where $a_{j}: \mathcal{S} \times \Theta \rightarrow \mathbb{R}_{+}$is known as the propensity function associated with the jump $\nu_{j}$. For any $\theta$, we define $a_{j}(x ; \theta)=0$ for those $x$ where $x+\nu_{j} \notin \mathbb{Z}_{+}^{d}$. The propensity functions depend on a parameter $\theta \in \Theta$, where $\Theta$ is assumed here to be finite dimensional.

The probability that the system jumps from $x \in \mathcal{S}$ to $x+\nu_{j} \in \mathcal{S}$ during the small interval $(t, t+d t)$ is

$$
\mathrm{P}\left(X(t+d t)=x+\nu_{j} \mid X(t)=x\right)=a_{j}(x ; \theta) d t+o(d t) .
$$

Example 2.1 (simple decay model). The simple decay model is a pure jump process $X$ in the lattice $\mathcal{S}=\Delta \mathbb{N}$, where $\Delta$ is a positive real number. The system starts from $x_{0} \in \mathcal{S}$ at time $t=0$, and the only reaction allowed is $\nu=-\Delta$. Its associated propensity function is $a(x ; c)=c x$, where $c>0$.

2.2. Upscaling: The mean field and Langevin equations. The generator $\mathcal{L}_{X}$ of a pure jump Markov process $X$ is a linear operator defined on the set of bounded functions. In our case, it is given by (see [10])

$$
\mathcal{L}_{X}(f):=\sum_{j} a_{j}(x ; \theta)\left(f\left(x+\nu_{j}\right)-f(x)\right) .
$$


Using a first-order Taylor expansion of $f$ in (2.1), we obtain the generator

$$
\mathcal{L}_{Z}(f):=\sum_{j} a_{j}(x ; \theta) \partial_{x} f(x) \nu_{j}
$$

which corresponds to the reaction-rates ODE (also known as the mean field ODE)

$$
\left\{\begin{array}{l}
d Z(t)=\nu a(Z(t) ; \theta) d t, t \in \mathbb{R}_{+}, \\
Z(0)=x_{0} \in \mathbb{R}_{+}
\end{array}\right.
$$

where the $j$-column of the matrix $\nu$ is $\nu_{j}$ and $a$ is a column vector with components $a_{j}$.

Using a second-order Taylor expansion of $f$ in (2.1), we obtain the generator of an Itô diffusion process, $Y$,

$$
\mathcal{L}_{Y}(f):=\sum_{j} a_{j}(x ; \theta)\left(\partial_{x} f(x) \nu_{j}+\frac{1}{2} \nu_{j}^{\top} \partial_{x}^{2} f(x) \nu_{j}\right),
$$

where $Y$ is the diffusion process defined by the Langevin Itô stochastic differential equation (SDE), where $B(t)$ is an $\mathbb{R}^{J}$ valued Wiener process with independent components

$$
\left\{\begin{array}{l}
d Y(t)=\nu a(Y(t) ; \theta) d t+\nu \operatorname{diag}(\sqrt{a(Y(t) ; \theta)}) d B(t), t \in \mathbb{R}_{+} \\
Y(0)=x_{0} \in \mathbb{R}_{+}
\end{array}\right.
$$

2.3. The second-order moment expansion. The mean field equations (2.2) approximate the evolution of the mean of the pure jump process $X$. But, sometimes, it is desirable to have ODEs that approximate higher-order moments as well. Here, we show how to derive a system of ODEs for the evolution of the first two moments of a pure jump process by approximating it with a Gaussian process. This approach is well suited for unimodal distributions of $X(t)$ for all times. In such a case, it has a main advantage with respect to the Langevin diffusion approach (2.3) because we do not need to sample any random variables, and in particular diffusion bridges, for obtaining estimations of our parameters.

Direct approach. Consider the Dynkin formula [10] for the process $X$,

$$
\mathrm{E}[f(X(t))]=f(X(0))+\int_{0}^{t} \mathrm{E}\left[\mathcal{L}_{X}(f)(s)\right] d s .
$$

To obtain the second-order moment expansion, we simply consider the formula (2.4) applied to $f(x)=x$ and $f(x)=x^{2}$. This leads to

$$
\left\{\begin{array}{l}
\mathrm{E}[X(t)]=x_{0}+\int_{0}^{t} \mathrm{E}\left[\sum_{j} a_{j}(X(s) ; \theta) \nu_{j}\right] d s, \\
\mathrm{E}\left[X^{2}(t)\right]=x_{0}^{2}+\int_{0}^{t} \mathrm{E}\left[\sum_{j} a_{j}(X(s) ; \theta)\left(2 \nu_{j} X(s)+\nu_{j}^{2}\right)\right] d s .
\end{array}\right.
$$

In general, the system (2.5) is not closed, and it depends on the form of the propensity functions $a_{j}$. In the linear case, when $a_{j}(x ; \theta)=g(\theta) x$, the system (2.5) is closed. We derive in section 4.2 an ODE system for $\mu(t):=\mathrm{E}[X(t)]$ and $\sigma^{2}(t):=$ $\mathrm{E}\left[(X(t)-\mu(t))^{2}\right]$.

An alternative approach. We present here an alternative way of deriving a system of ODEs that approximately describes the evolution of the first two moments 
of the process $X$. The advantage of this approach is that it always gives a closed system of ODEs.

Consider a general SDE of the form

$$
d Y(t)=\alpha(Y(t)) d t+\beta(Y(t)) d B(t) .
$$

It is possible to approximate the moments of $Y(t)$ as follows. First, take expectations on both sides of (2.6) and on both sides of the SDE, which results from applying the Itô formula to the function $g(y, t):=(y-\mathrm{E}[Y(t)])^{2}$. Then, define $\delta(t):=Y(t)-\mathrm{E}[Y(t)]$ and Taylor-expand $\alpha(Y(t)), \beta(Y(t))$ and $\delta(t)(\alpha(t)-\mathrm{E}[\alpha(t)])$ around $\mathrm{E}[Y(t)]$ in powers of $\delta(t)$. Finally, drop the terms of order $\mathrm{E}\left[\delta^{3}(t)\right]$ and higher. In that way, we obtain the following system of ODEs:

$$
\begin{cases}d \mu(t) & =\left(\alpha(\mu(t))+\alpha^{\prime \prime}(\mu(t)) \sigma(t) / 2\right) d t, \\ d \sigma^{2}(t) & =\left(\left(2 \alpha^{\prime}(\mu(t))+\gamma^{\prime \prime}(\mu(t))\right) \sigma^{2}(t)+2 \gamma(\mu(t))\right) d t, \\ \left(\mu(0), \sigma^{2}(0)\right) & =\left(x_{0}, 0\right), x_{0} \in \mathbb{R}_{+}, t \in \mathbb{R}_{+},\end{cases}
$$

where $\gamma(y):=\beta(y)^{2} / 2$ such that $\mu(t)$ and $\sigma^{2}(t)$ approximate $\mathrm{E}[Y(t)]$ and $\mathrm{E}\left[\delta^{2}(t)\right]$, respectively. This moment expansion approach can be extended directly to the multidimensional case. For the linear case, when $a_{j}(x ; \theta)=g(\theta) x$, the system in $(2.7)$ is equivalent to the system in (2.5).

Remark 2.2. When the distribution of $X(t)$ is multimodal, then we can extend the approach in (2.7) by approximating the distribution of $X(t)$ with a Gaussian mixture. The price to pay in this case is the increment in the dimension of the resulting ODE system.

3. The thickness measurement process. In this section, we define an auxiliary process called the thickness process that is used for modeling and inference convenience. The relation between the wearing and the thickness processes is simple: the sum of both is a constant that equals the initial thickness. Therefore, the thickness process is decreasing and takes positive values in the lattice generated by caliper precision.

It is worth mentioning that the simple decay model described in Example 2.1 predicts a much smaller variability than does the one observed in the data, and it cannot be used as a model for the thickness process. For that reason, we propose modeling the thickness process using two reactions with linear state-dependent coefficients. This model generates satisfactory confidence bands that we use as a goodness-of-fit test.

Definition of the thickness process. Let $X(t)$ be the thickness process derived from the wear of the cylinder liners up to time $t$ (see [7, 8]), i.e., $X(t)=T_{0}-W(t)$, where $W$ is the wear process and $T_{0}$ is the initial thickness. We model $X(t)$ as a sum of two simple decay processes (see Example 2.1) with $\Delta=0.05$ (which is the resolution of the measurement instrument), since one simple decay process is not enough to explain the variance of the data. The two considered intensity-jump pairs are $\left(a_{1}(x), \nu_{1}\right)=\left(c_{1} x,-\Delta\right)$ and $\left(a_{2}(x), \nu_{2}\right)=\left(c_{2} x,-k \Delta\right)$, where $k$ is a positive integer to be determined, and $c_{1}$ and $c_{2}$ are coefficients with dimension $(\mathrm{mm} \cdot \mathrm{hour})^{-1}$. Therefore, the probability of observing a thickness decrement in a small time interval $(t, t+d t)$ is

$$
\begin{gathered}
P(X(t+d t)=X(t)-\Delta \mid X(t)=x)=c_{1} x d t, \\
P(X(t+d t)=X(t)-k \Delta \mid X(t)=x)=c_{2} x d t,
\end{gathered}
$$

Copyright $@$ by SIAM. Unauthorized reproduction of this article is prohibited. 
where the initial thickness $X(0)=T_{0}$, and the coefficients $c_{1}, c_{2}$, and $k$ are four unknown parameters.

4. Inference for the thickness measurement process. In this section, we obtain the likelihood functions at the macroscopic level for the thickness process (3.1). The first step is to transform the data set to observe a decreasing thickness process. We define the thickness data, $\mathbf{x}=\left\{x_{i}\right\}_{i=1}^{n}$, as $x_{i}:=T_{0}-w_{i}$, where $T_{0}$ is an unknown parameter that we expect to be around $5.0 \mathrm{~mm}$ (see section 6 for further details) and $w_{i}$ is the wear of the $i$ th datum. Observe that $\mathbf{x}$ depends on $T_{0}$.

We consider two approximate models for the experimental data, $\mathbf{x}$, as follows. The first postulates that each data point is the mean field of the thickness process plus Gaussian noise with constant variance. In this case, the maximum likelihood estimation (MLE) leads to an ordinary least squares problem. This model turns out to be unsatisfactory for two reasons: when we consider only one reaction, it gives a very narrow confidence band; when we consider two reactions, there is an identifiability problem since there is a straight line in which the maximum of the likelihood is attained. The second is slightly more complex. It postulates that the data are the sum of two terms that are independent realizations of two Gaussian random variables. The moments of the first random variable evolve in time according to a system of ODEs obtained by moment expansion. The second term is just additive Gaussian noise with constant variance. The MLE leads to a weighted least squares problem with a logarithmic penalization term. In this case, as we see in section 6 , there is only one point in which the maximum of the likelihood is attained.

4.1. Mean field approximation. Let us consider a mean field approximation for the thickness data, $\mathbf{x}$; i.e., the data $\mathbf{x}$ are modeled according to

$$
x_{i}=Z\left(t_{i}\right)+\epsilon_{i} \quad(\text { model } 1),
$$

where $Z(t)$ satisfies the mean field ODE (2.2) and $\epsilon_{i}$ are independent and identically distributed (i.i.d.) realizations of $\mathcal{N}\left(0, \sigma_{E}^{2}\right)$ for $i=1, \ldots, n$, where $\sigma_{E}^{2}>0$ is the experimental measurement error. In this work, we set $\sigma_{E}=\Delta$ (see Remark 4.1).

In this case, the likelihood function can be written as

$$
L(\theta ; \mathbf{x}) \propto \prod_{i=1}^{n} \exp \left\{-\frac{\left(x_{i}-Z\left(t_{i} ; \theta\right)\right)^{2}}{2 \sigma_{E}^{2}}\right\},
$$

where $\theta=\left(c_{1}, c_{2}, k, T_{0}\right)$.

Now, given $k$ and $T_{0}$, the MLE for $\left(c_{1}, c_{2}\right)$ is the minimizer of the opposite of the $\log$ likelihood, i.e.,

$$
c^{*}\left(k, T_{0}\right):=\arg \min _{c_{1} \geq 0, c_{2} \geq 0} \sum_{i=1}^{n}\left(x_{i}-Z\left(t_{i} ; \theta\right)\right)^{2} .
$$

4.2. A Gaussian approximation based on moment expansion. To obtain a system of ODEs for the time evolution of the first two moments of the process $X$, we proceed to write the system (2.5) in a differential form, where the propensity functions and jumps are defined as in (3.1). Since the propensity functions are linear functions of the state, we have a closed system of ODEs:

$$
\begin{cases}d \mu(t) & =\left(c_{1} \nu_{1}+c_{2} \nu_{2}\right) \mu(t) d t \\ d \sigma^{2}(t) & =\left(2\left(c_{1} \nu_{1}+c_{2} \nu_{2}\right) \sigma^{2}(t)+\left(c_{1} \nu_{1}^{2}+c_{2} \nu_{2}^{2}\right) \mu(t)\right) d t \\ \left(\mu(0), \sigma^{2}(0)\right) & =\left(x_{0}, 0\right), x_{0} \in \mathbb{R}_{+}, t \in \mathbb{R}_{+}\end{cases}
$$


Its solution is given by

$$
\begin{aligned}
\mu(t) & =x_{0} \exp \left(\left(c_{1} \nu_{1}+c_{2} \nu_{2}\right) t\right), \\
\sigma^{2}(t) & =x_{0} \frac{c_{1} \nu_{1}^{2}+c_{2} \nu_{2}^{2}}{c_{1} \nu_{1}+c_{2} \nu_{2}} \exp \left(\left(c_{1} \nu_{1}+c_{2} \nu_{2}\right) t\right)\left(\exp \left(\left(c_{1} \nu_{1}+c_{2} \nu_{2}\right) t\right)-1\right) .
\end{aligned}
$$

Based on $\mu(t)$ and $\sigma^{2}(t)$, we consider a Gaussian model for our data; i.e., the data $\mathbf{x}$ are modeled according to

$$
x_{i}=\tilde{Y}\left(t_{i}\right)+\epsilon_{i} \quad(\text { model } 2),
$$

where $\tilde{Y}(t) \sim \mathcal{N}\left(\mu(t), \sigma^{2}(t)\right)$, with mean $\mu(t)$ and variance $\sigma^{2}(t)$. We also consider that $\epsilon_{i}$ are i.i.d. realizations of $\mathcal{N}\left(0, \sigma_{E}^{2}\right)$ for $i=1, \ldots, n$, where $\sigma_{E}^{2}>0$ is the experimental measurement error. Here, $\sigma_{E}=\Delta$ (see Remark 4.1).

In this case, the likelihood function can be written as

$$
L(\theta ; \mathbf{x})=\prod_{i=1}^{n} \frac{1}{\sqrt{2 \pi\left(\sigma_{E}^{2}+\sigma^{2}\left(t_{i} ; \theta\right)\right)}} \exp \left\{-\frac{\left(x_{i}-\mu\left(t_{i} ; \theta\right)\right)^{2}}{2\left(\sigma_{E}^{2}+\sigma^{2}\left(t_{i} ; \theta\right)\right)}\right\}
$$

where $\theta=\left(c_{1}, c_{2}, k, T_{0}\right)$.

Now, the MLE for $\left(c_{1}, c_{2}\right)$, for fixed $k$ and $T_{0}$, is the minimizer of the opposite of the log likelihood,

$$
c^{*}\left(k, T_{0}\right):=\arg \min _{c_{1} \geq 0, c_{2} \geq 0} \sum_{i=1}^{n}\left\{\frac{\left(x_{i}-\mu\left(t_{i} ; \theta\right)\right)^{2}}{\sigma_{E}^{2}+\sigma^{2}\left(t_{i} ; \theta\right)}+\log \left(\sigma_{E}^{2}+\sigma^{2}\left(t_{i} ; \theta\right)\right)\right\} .
$$

Finally, we determine the appropriate values of $k$ and $T_{0}$ by analyzing the sequence $\left\{c^{*}\left(k, T_{0}\right)\right\}_{k \geq 2}$ for different values of $T_{0}$. In section 6 , we see that for our data set, $\mathbf{w}$, the appropriate values for $k$ and $T_{0}$ are 4 and $5.0 \mathrm{~mm}$, respectively.

Remark 4.1. Since the precision of the caliper is $\Delta$, if we assume that the measurement errors are normally distributed, then the interval $\pm \Delta / 2$ is approximately $\pm 3 \sigma_{E}$ wide, and therefore we could set $\sigma_{E}=2 \Delta / 3$. Numerical experiments show that our inferences are essentially the same whether $\sigma_{E}$ is $\Delta$ or $2 \Delta / 3$.

Remark 4.2. From the Langevin equation (2.3), if we define $\alpha(y):=\nu a(y ; \theta)$ and $\beta(y):=\nu \operatorname{diag}(\sqrt{a(y ; \theta)})$, we again obtain the system in (4.4).

Remark 4.3. The wear process, by its physical nature, is increasing and bounded, and therefore the thickness process should be decreasing and bounded from below. Thus, we expect that the mean of the thickness process, $\mu(t)$, defined in (4.5), decays to zero. Regarding the variance, $\sigma^{2}(t)$, defined in (4.6), it should start from zero at time zero, increase, and then return to zero again.

5. Hitting times. In this section, we address the problem of computing the distribution of the time in which the wear attains a certain critical value, $L$, i.e., the hitting time to $L$. Let $\tau_{L}$ be the first time that the wear process, $W$, is greater than or equal to the critical level, $L$,

$$
\tau_{L}:=\inf \left\{t \in \mathbb{R}_{+}: W(t) \geq L\right\} .
$$

This is exactly the first time that the thickness process, $X$, is less than or equal to $B=T_{0}-L$, where $T_{0}$ is the initial thickness.

We have that $F_{\tau_{B} ; \theta}(t):=\mathrm{P}(X(t) \leq B \mid \theta)=\sum_{x \leq B} p_{x}(t ; \theta)$, where $p_{x}(t ; \theta)$ is the probability that $X(t)=x$, given the value of the parameter vector $\theta$. We know that $p_{x}(t ; \theta)$ satisfies a system of ODEs called the Master Equation (ME) (see $\left.[3,12,13]\right)$. 
In our setting, the ME is given by

$$
\left\{\begin{aligned}
\frac{d p_{x}(t ; \theta)}{d t} & =\sum_{j} p_{x-\nu_{j}}(t ; \theta) a_{j}\left(x-\nu_{j} ; \theta\right)-p_{x}(t ; \theta) \sum_{j} a_{j}(x ; \theta), t \in \mathbb{R}_{+}, \\
p_{x}(0 ; \theta) & =\mathbf{1}_{\left\{x=x_{0}\right\}},
\end{aligned}\right.
$$

where $\mathbf{1}_{A}$ is the indicator function of the set $A, x, x+\nu_{j} \in \mathcal{S}$, and $\theta=\left(c_{1}, c_{2}, k, T_{0}\right)$. This system of ODEs can be efficiently solved by any standard numerical technique.

5.1. Conditional residual reliability. Suppose that we know that the wear process, $W$, is at level $w_{0}$ at time $t_{0} \geq 0$. Assume that there exists a critical stopping level, $w_{\max }>w_{0}$, that determines the residual lifetime $\tau_{\max }-t_{0}$. For $t>0$, the residual lifetime is greater than $t$ if and only if $W\left(t_{0}+t\right)<w_{\max }$. Therefore, the conditional probability

$$
\mathrm{P}\left(\tau_{\max }-t_{0}>t \mid W\left(t_{0}\right)=w_{0}\right)=\mathrm{P}\left(W\left(t_{0}+t\right)<w_{\max } \mid W\left(t_{0}\right)=w_{0}\right) .
$$

Taking into account the relation between the wear and the thickness processes, we have that the conditional residual reliability function defined as

$$
R\left(t ; t_{0}, w_{0}\right):=\mathrm{P}\left(\tau_{\max }-t_{0}>t \mid W\left(t_{0}\right)=w_{0}\right)
$$

can be written as $\mathrm{P}\left(X\left(t ; T_{0}-w_{0}\right)>T_{0}-w_{\max }\right)$, where $X\left(\cdot, x_{0}\right)$ is the thickness process starting from $x_{0}$.

6. Numerical results. As mentioned at the beginning of the section 3 , simple decay models (see Example 2.1) do not fit the wear data, $\mathbf{w}=\left\{w_{i}\right\}_{i=1}^{n}$, since they produce very narrow confidence bands, like the inner dashed (blue) lines shown in the left panel of Figure 4. In fact, we modeled $a(x ; \theta)$ as $\sum_{j=1}^{J} c_{j} x^{j}$ for $J=1,2,3$. In each case the only nontrivial coefficient was $c_{1}$. It is important to notice that all the confidence bands are computed using the ME (5.1).

Consider the pure jump process defined in (3.1). For this process, we have to estimate the values of $c_{1}, c_{2}, k$, and $T_{0}$. Figure 2 shows, in the left panel, the contour plot of the least squares function (4.3), associated with the likelihood function defined in (4.2), for $k=4$ (for other values of $k$, we obtain the same results) and $T_{0}=5.0$. We can observe an identifiability problem since the maximum of the likelihood function is attained at a straight line; see the left panel of Figure 2. By varying the values of $c_{1}$ and $c_{2}$ in the minimum level set of the least squares function, we obtain a family of confidence bands. For $c_{2}=0$ (one reaction model) or small values of $c_{2}$, the confidence band is very narrow; see the right panel of Figure 2. At the other extreme, when $c_{1}$ is positive but close to zero, we obtain satisfactorily wide confidence bands, shown in the right panel of Figure 3.

To properly identify the values of $c_{1}$ and $c_{2}$ for each integer $k \geq 2$ and $T_{0} \in \mathcal{S}$, we use model 2 , defined in (4.7), for the thickness data $\mathbf{x}$. Figure 3 shows, in the left panel, the contour plot of the least squares function (4.9), associated with the likelihood function defined in (4.8) for $k=4$ and $T_{0}=5.0$. Now, we are in a better situation regarding identifiability. Conditional on those values of $k$ and $T_{0}$, the MLE for $\left(c_{1}^{*}, c_{2}^{*}\right)$ is given by $\left(0.63 \cdot 10^{-4}, 1.2 \cdot 10^{-4}\right)$. In the right panel, the corresponding $90 \%$ confidence band is shown, which is very similar to the one obtained in [8], but we use a more parsimonious model for the wearing process.

Figure 4 shows in the left panel the wear data, w, along with the confidence bands computed for the data models 1 and 2. The values of $c_{1}, c_{2}$, and $k$ were 

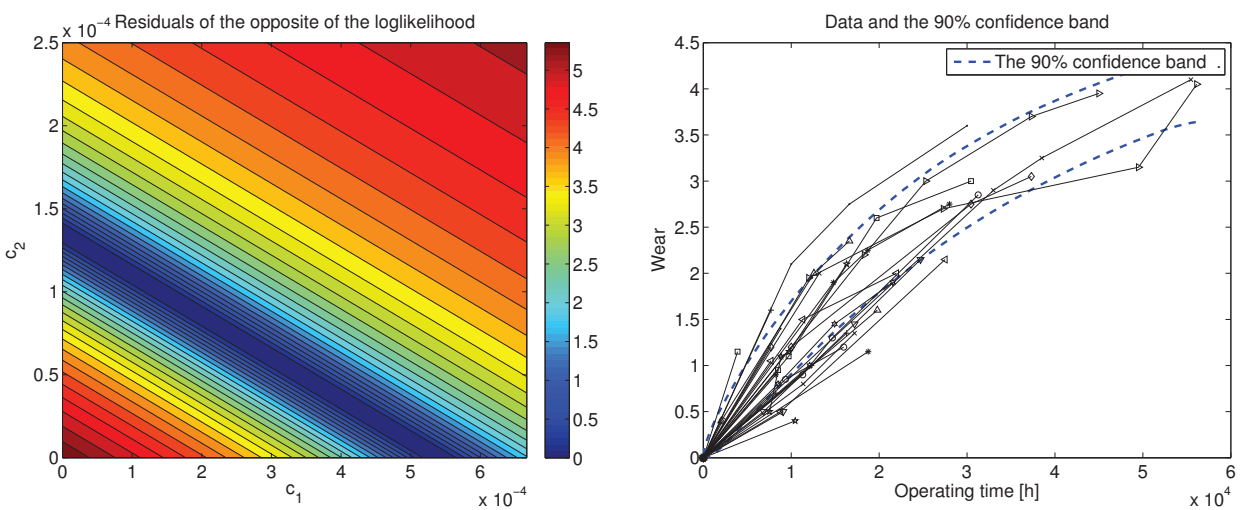

FIG. 2. Left panel: residuals of the opposite of the loglikelihood of (4.2) for $k=4$. There is an identifiability problem for the parameters $c_{1}$ and $c_{2}$. For each pair in the minimizing set, we have a different confidence band. Right panel: wear data and the $90 \%$ confidence band under model 1 , defined in (4.1), for positive but small $c_{2}$. The confidence band turns out to be narrow when $c_{1}$ increases.
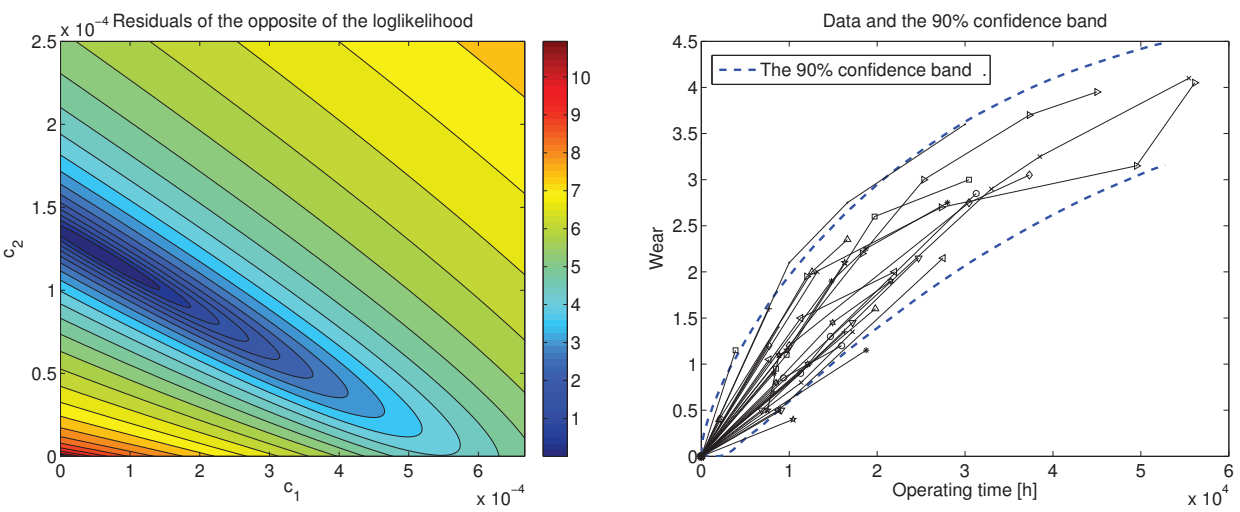

FIG. 3. Left panel: residuals of the minus loglikelihood (4.8) for $k=4$. Model 2, defined in (4.7), for the thickness data, $\mathbf{x}$, produces a likelihood function with a unique global maximum. The MLE is $\left(c_{1}^{*}, c_{2}^{*}\right)=\left(0.63 \cdot 10^{-4}, 1.2 \cdot 10^{-4}\right)$. Right panel: the $90 \%$ confidence band.

computed using the upscaled models, but fitted using the ME, which acts directly in the microscopic base model (3.1).

Now, consider the values of the objective function defined in (4.9), evaluated at $\theta^{*}:=\left(c^{*}\left(k, T_{0}\right), k, T_{0}\right)$, as a function of $k$ and $T_{0}$,

$$
F\left(k, T_{0}\right):=\sum_{i=1}^{n}\left\{\frac{\left(x_{i}-m\left(t_{i} ; \theta^{*}\right)\right)^{2}}{\sigma_{E}^{2}+\sigma^{2}\left(t_{i} ; \theta^{*}\right)}+\log \left(\sigma_{E}^{2}+\sigma^{2}\left(t_{i} ; \theta^{*}\right)\right)\right\} .
$$

Figure 4 shows in the right panel that $F\left(k, T_{0}=5.0 \mathrm{~mm}\right)$ decreases until $k=4$, where it reaches a plateau. The same situation is true for other values of $T_{0}$, even more, $F(4,5.0) \leq F\left(4, T_{0}\right)$ for $T_{0} \in \mathcal{S}$. For that reason, $\theta^{* *}:=\left(0.63 \cdot 10^{-4}, 1.2 \cdot 10^{-4}, 4,5.0\right)$ is the MLE for our model. As a consequence, we identify two types of jumps: one with amplitude $\Delta$ and the other with amplitude $4 \Delta$.

Figure 5 shows the evolution in time of the probability mass function defined in (5.1), $p_{x}\left(t ; \theta^{* *}\right)$, which is the solution of an ODE system. It looks like the typical 

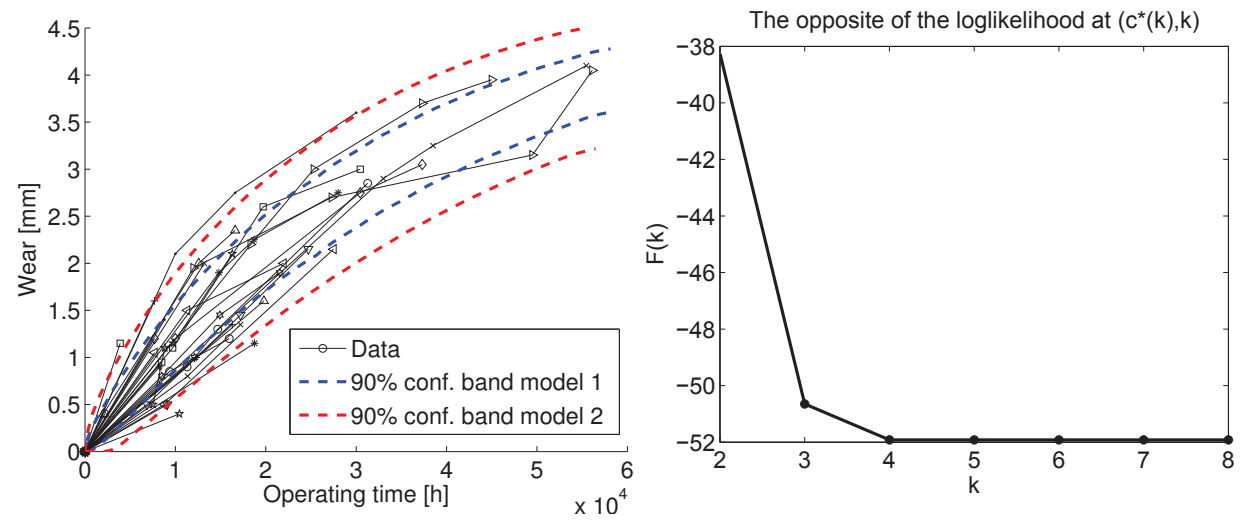

FIG. 4. Left panel: the exact $90 \%$ confidence band from the ME (5.1). Right panel: plot of $F(k)$ defined in (6.1). $F(k)$ decreases until $k=4$, where it reaches a plateau.

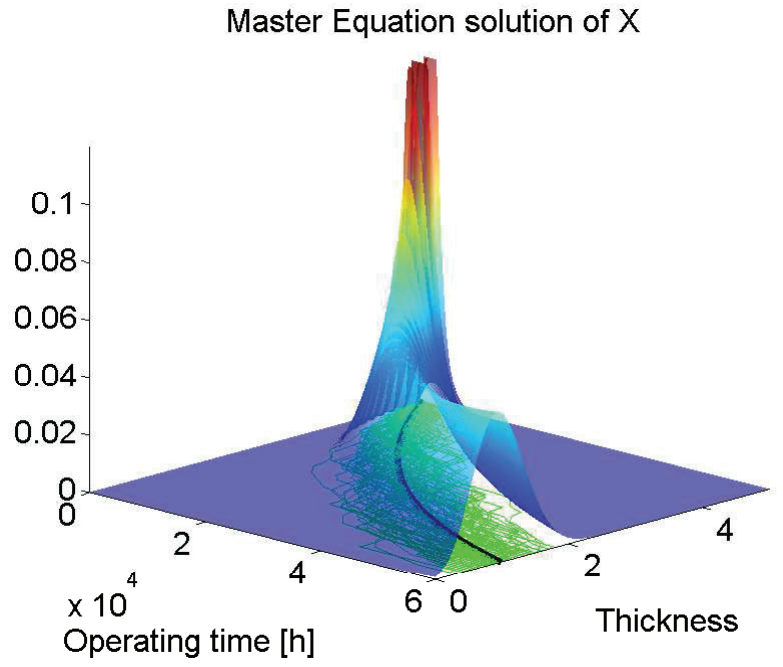

FIG. 5. Solution of the ME (5.1) and 100 exact simulated paths [4].

surface obtained in the Fokker-Planck equations for diffusions, but this is because we are considering a fine lattice, $\mathcal{S}=\Delta \mathbb{N}$, with $\Delta=0.05$. We see that it departs at time $t=0$ from a point mass concentrated at the initial thickness $T_{0}$, and it diffuses into a unimodal bell-shaped distribution. In the domain, we plotted 100 exact simulated paths and their average (see [4]).

In section 5, we defined the hitting time to the critical level $L$. Let $L=4$, as specified in warranty clauses. Then, since we have $T_{0}=5.0$, we have that $B=1$. We can see the cumulative distribution function $(\mathrm{CDF})$ and the probability distribution function (PDF) of the hitting time, $\tau_{B}$, for $B=1$, in the left and right panels of Figure 6 , respectively. The figure indicates that at around $t=30,000$ hours, it is advisable to start monitoring the wear.

Figure 7, in the left panel, shows the evolution of the Gaussian confidence intervals with the mean and variance computed from the process $X$. The functions $\mu(t)$ and $\sigma^{2}(t)$ are defined in (4.4). In the right panel of Figure 7, we see that the confidence 

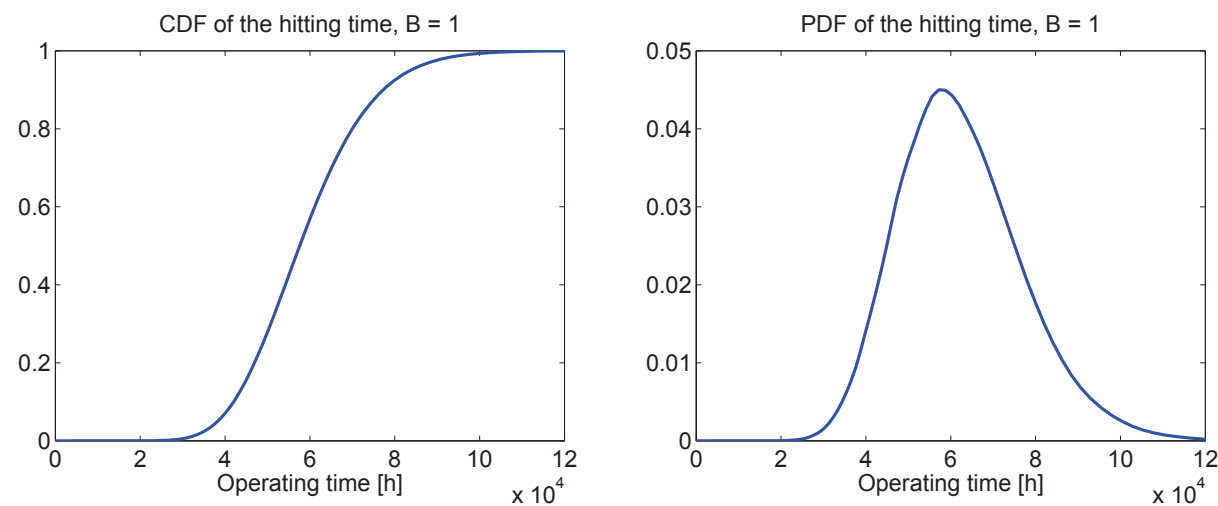

FIG. 6. Left panel: $C D F$ of the hitting time for $B=1$. Right panel: PDF of the hitting time to the critical level.
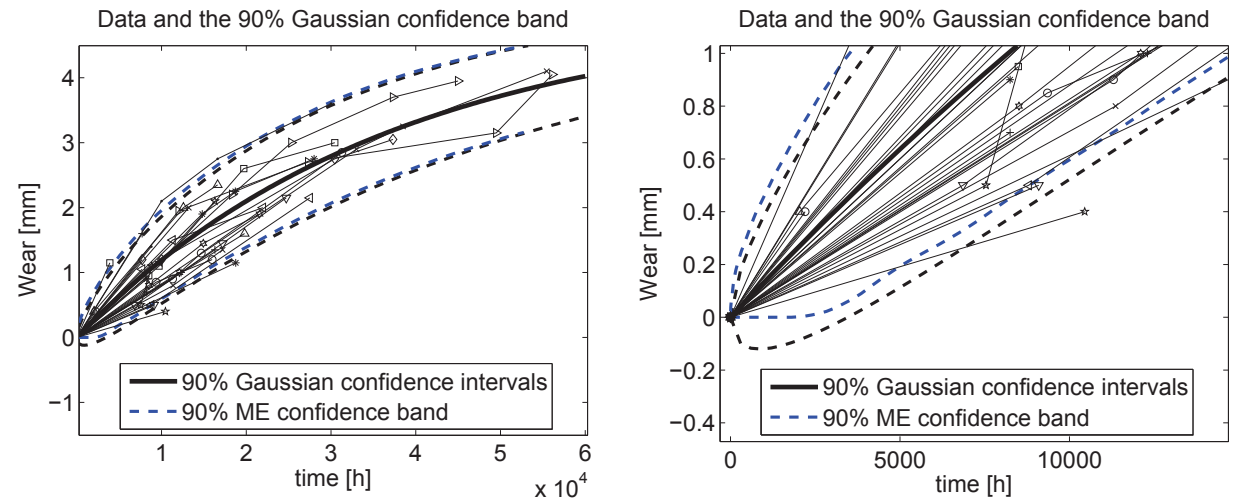

FIG. 7. Left panel: wear data and the band made from the $90 \%$ confidence intervals $\mu(t) \pm$ $1.645 \sqrt{\sigma^{2}(t)+\sigma_{E}^{2}}$, where $\mu(t)$ and $\sigma^{2}(t)$ are defined as in (4.4). In this case, $\mu(t)$ and $\sigma^{2}(t)$ describe exactly the evolution of the mean and variance of the process $X$. Right panel: details of the left panel. We see that the confidence band computed from the $M E$ (5.1) does not contain any negative value.

band computed from the ME (5.1) does not contain any negative value.

In Figure 8, we show in the left panel the QQ-plot of the normalized thickness data, $\mathbf{z}=\left\{z_{i}\right\}_{i=1}^{n}$, defined by $z_{i}=\left(x_{i}-\mu\left(t_{i}\right)\right) / \sqrt{\sigma^{2}\left(t_{i}\right)+\sigma_{E}^{2}}$, where the thickness data $\left\{x_{i}\right\}_{i=1}^{n}$ are defined as in section 4 . The figure suggests that there is good agreement between $\mathbf{z}$ and the standard Gaussian distribution. In the right panel of Figure 8, we show the percentage histogram and a kernel density estimation of $\mathbf{z}$. The p-value of the Shapiro-Wilk test is 0.68 . We therefore cannot reject Gaussianity. This analysis strongly supports the use we made of model 2, defined in (4.7), for the thickness data, $\mathrm{x}$.

Figure 9 shows behavior of the conditional residual reliability function, $R\left(t ; 0, w_{0}\right)$ (see section 5.1) for some values of $w_{0}$. In this case, we set $w_{\max }=4$. As expected, for a fixed residual lifetime $t$, we have that $R\left(t ; 0, w_{0}\right)$ is a decreasing function of $w_{0}$.

7. Conclusions. In this paper, we presented a novel approach to the problem of modeling the wear process of cylinder liners. Since the measuring caliper has finite precision, the wear process takes values in a lattice, and therefore a pure jump 

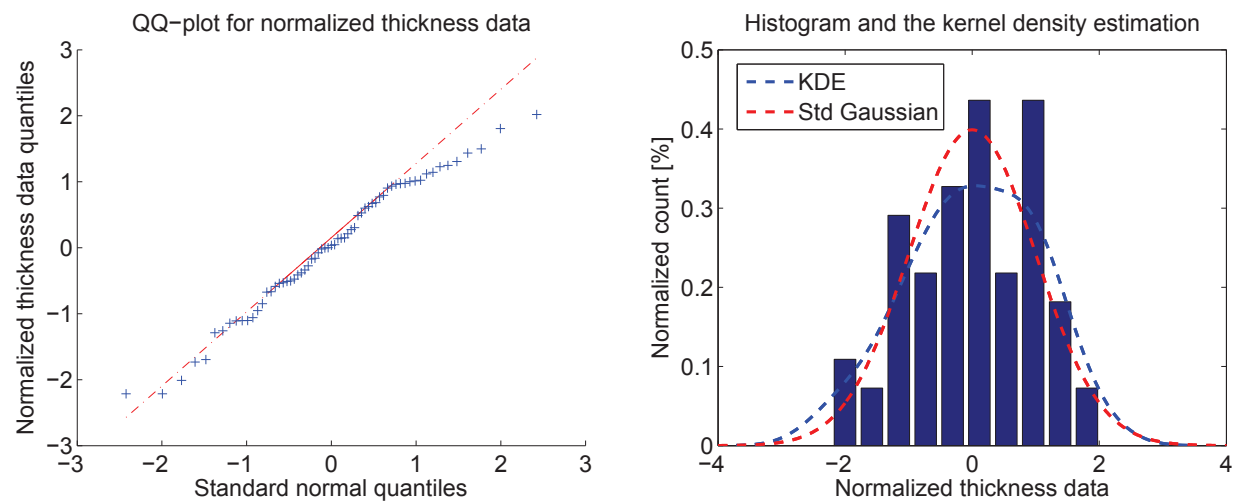

FIG. 8. Left panel: $Q Q$-plot of the normalized thickness data, $z_{i}=\left(x_{i}-\mu\left(t_{i}\right)\right) / \sqrt{\sigma^{2}\left(t_{i}\right)+\sigma_{E}^{2}}$. This plot suggests that there is good agreement between $\mathbf{z}$ and the standard Gaussian distribution. Right panel: percentage histogram and a kernel density estimation of $\mathbf{z}$. The p-value of the ShapiroWilk test is 0.68. Therefore we cannot reject Gaussianity.

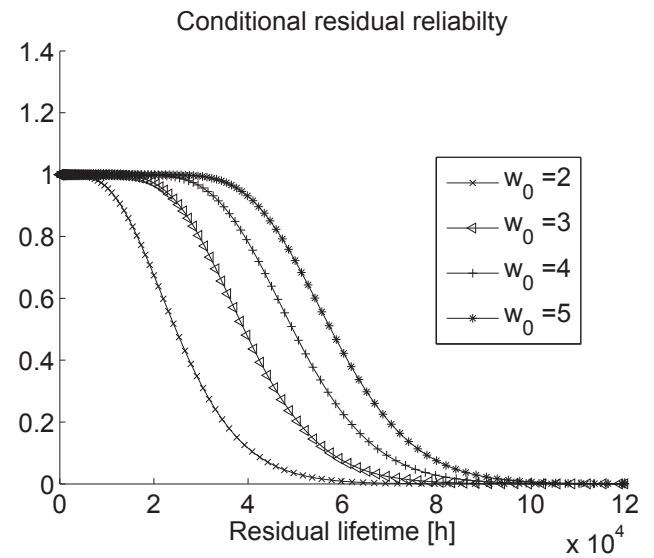

FIG. 9. The conditional residual reliability function, $R\left(t ; 0, w_{0}\right)$ (see section 5.1 ), for some values of $w_{0}$.

process is a sensible model. In this approach, we started fitting one of the most simple pure jump processes, i.e., the simple decay model, and added complexity only when necessary. We found that the wear process can be modeled using only two jumps of amplitudes, $\Delta$ and $4 \Delta$, with linear propensity functions. In contrast to the work of Giorgio, Guida, and Pulcini [8], we did not need to use age-dependent propensity functions or gamma noise. Nevertheless, our approach is totally suitable for dealing with age-dependent propensities since time does not play any other role than a given constant.

One of the main contributions of this work is the multiscale indirect inference approach, where the inferences are based on upscaled models. The coefficients of the linear propensity functions were inferred using the likelihood associated with a Gaussian upscaled model. The mean and variance of this Gaussian process are the solutions of a second-order moment expansion ODE system. In this way, we computed the MLE by solving a standard nonlinear least squares problem. We observe that this method is much simpler than dealing directly with the likelihood of the 
pure jump process, which in general cannot be expressed in closed form and requires computationally intensive sampling techniques to be solved. We notice that, as long as the probability distribution of the pure jump process is unimodal at every time, our Gaussian inference approach is applicable and produces substantial savings in the computational work. Otherwise, the Langevin model, while more computationally demanding, is more flexible.

Thanks to the remarkable simplicity of our model, we can easily obtain the distribution of any observable of the process directly from the solution of the ME, which provides the probability distribution of the process at all times. From this probability mass function, we easily compute the CDF of the hitting time to the critical value stipulated in the warranty and the conditional residual reliability function. It is worth mentioning that we did not use Monte Carlo simulation or any other sampling procedure.

\section{REFERENCES}

[1] C. Bayer and J. Schoenmakers, Simulation of Conditional Diffusions via Forward-Reverse Stochastic Representations, preprint, arXiv:1306.2452, 2013.

[2] M. Bladt And M. Sørensen, Statistical inference for discretely observed Markov jump processes, J. Roy. Statist. Soc. Ser. B, 67 (2005), pp. 395-410.

[3] C. Gardiner, Stochastic Methods: A Handbook for the Natural and Social Sciences, 4th ed., Springer Ser. Synergetics, Springer-Verlag, Berlin, 2009.

[4] D. T. Gillespie, A general method for numerically simulating the stochastic time evolution of coupled chemical reactions, J. Comput. Phys., 22 (1976), pp. 403-434.

[5] M. Giorgio, M. Guida, And G. Pulcini, A wear model for assessing the reliability of cylinder liners in marine diesel engines, IEEE Trans. Reliability, 56 (2007), pp. 158-166.

[6] M. Giongio, M. Guida, And G. Pulcini, Stochastic processes for modeling the wear of marine engine cylinder liners, in Statistics for Innovation, Pasquale Erto, ed., Springer, Milan, 2009, pp. 213-230.

[7] M. Giorgio, M. Guida, And G. Pulcini, A state-dependent wear model with an application to marine engine cylinder liners, Technometrics, 52 (2010), pp. 172-187.

[8] M. Giorgio, M. Guida, And G. Pulcini, An age- and state-dependent Markov model for degradation processes, IIE Trans., 43 (2011), pp. 621-632.

[9] C. Gourieroux, A. Monfort, and E. Renault, Indirect inference, J. Appl. Econometrics, 8 (suppl.), (1993), pp. S85-S118.

[10] F. Klebaner, Introduction to Stochastic Calculus with Applications, 3rd ed., Imperial College Press, London, 2012.

[11] J. Norris, Markov Chains, Cambridge Ser. Statist. Probab. Math. 7, Cambridge University Press, Cambridge, UK, 1998.

[12] H. Risken And T. Frank, The Fokker-Planck Equation: Methods of Solution and Applications, Springer Ser. Synergetics, Springer-Verlag, Berlin, 1996.

[13] N. Van Kampen, Stochastic Processes in Physics and Chemistry, 3rd ed., North-Holland Personal Library, Elsevier, Amsterdam, 2007.

[14] D. J. Wilkinson, Stochastic Modelling for Systems Biology, 2nd ed., Chapman \& Hall/CRC Math. Comput. Biol. Ser., Chapman \& Hall/CRC Press, Boca Raton, FL, 2012. 